\title{
Diseño de recurso educativo abierto con un sistema experto para la enseñanza-aprendizaje de base de datos
}

\author{
Erick Nila Méndez, Cristina Juárez Landín, José Luis Sánchez Ramírez, \\ Anabelem Soberanes Martin \\ Universidad Autónoma del Estado de México, Maestría en Ciencias de la \\ Computación, C. U. Valle de Chalco, Estado de México, \\ México \\ li.ericknm@live.com.mx, cjuarezl@uaemex.mx, jluissar@gmail.com, \\ belemsoberanes@yahoo.com.mx
}

\begin{abstract}
Resumen. En este artículo se presenta el desarrollo de Recursos Educativos Abiertos (REA) por medio de un Sistema Experto (SE) como complemento al proceso de enseñanza-aprendizaje, y así cumplir con los requisitos principales para facilitar su adición a repositorios de REA. El proceso de desarrollo se basa en el Modelo tecnológico para la creación de un REA propuesto en 2014. Dichos recursos apoyan el aprendizaje y la práctica docente aumentando el acervo de conocimientos y recursos disponibles de forma libre a la comunidad estudiantil. Del mismo modo se extiende la labor del docente fuera del aula complementando y apoyando el aprendizaje del alumno de dos formas, la primera con un REA que cumple con todos los requisitos estandarizados de desarrollo y la segunda con un Sistema Experto que interactúa con el estudiante como si estuviera con el docente de forma presencial evaluando su aprendizaje.
\end{abstract}

Palabras clave: Recurso educativo abierto, sistema experto, repositorio, enseñanza, aprendizaje, inteligencia artificial.

\section{Design of Open Educational Resource with an Expert System for Teaching-Learning of Data Bases}

\begin{abstract}
This article describes the development of Open Educational Resources (OER) through an expert system (SE) to supplement the teaching-learning process, and thus fulfill the main requirements for easy addition to OER repositories. The development process is based on the technological model for the creation of OER proposed in 2014. These resources support learning and teaching practice increasing the wealth of knowledge and resources freely available to the student community. Similarly the work of teachers outside the classroom extends complementing and supporting student learning in two ways, first with an OER that meets all standard requirements of development and the second with an expert system that interacts with the student as if I were with the teacher in person evaluating their learning.
\end{abstract}

Keywords: Open educational resource, expert system, repository, teaching, learning, artificial intelligence. 


\section{Introducción}

La UNESCO [1] menciona que el acceso universal a la educación de calidad contribuye al desarrollo sostenible de la sociedad y de la economía.

El rol de la tecnología en su rápido y cambiante crecimiento demanda respuestas ágiles en el campo educativo, tanto por parte de las instituciones como del personal docente, en la medida en que se implementan tecnologías educativas que enriquecen los ambientes de aprendizaje en el aula [2].

El aumento del acceso a Internet ha promovido aún más el estudio individualizado y en conjunto con las redes sociales y el aprendizaje colaborativo ha generado oportunidades para la innovación pedagógica.

Una gran cantidad de recursos educativos, producto del avance tecnológico, están ahora al alcance de las aulas. Además, a esta evolución se ha sumado una nueva tendencia hacia la apertura y la democratización del conocimiento, y ha dado lugar al movimiento de los recursos abiertos. Con esto surgen como una innovación en la educación los Recursos Educativos Abiertos (REA) [3].

Es de suma importancia la creación e implementación de los REA para impulsar el libre aprendizaje de los alumnos como también apoyar la práctica docente. Sin embargo hay que considerar que no todos los REA existentes son o fueron creados bajo un estándar, además que se considera de manera manifiesta la presencia de los docentes y en consecuencia los alumnos reducen su opción de estudio más allá del aula.

Por lo anterior, es un motivo que impulsa esta investigación y que en conjunto con un Sistema Experto (SE) que complementa al REA para obtener recursos computacionales para la enseñanza y aprendizaje contribuyendo a la línea de Inteligencia Artificial en la educación.

El Sistema Experto hace que el docente este de manera virtual complementando la enseñanza-aprendizaje más allá del aula por lo que el alumno tiene una retroalimentación del tema.

\subsection{Recursos Educativos Abiertos (REA)}

Los REA son materiales de enseñanza, aprendizaje e investigación, en este caso en soporte digital que son de dominio público o con una licencia abierta lo que permite el acceso gratuito a estos materiales los cuales pueden ser usados, adaptados $\mathrm{y}$ redistribuidos.

En 2001, el MIT (Massachusetts Institute of Tecnology) anunció la publicación de sus cursos en Internet para que fueran accesibles a todo el público, lo que marco el precedente para que la UNESCO en el 2002, en el primer foro mundial sobre recursos educativos de libre acceso definiera el término "recursos educativos de libre acceso" [1].

A partir de ese momento todas las instituciones educativas deben de contar con un repositorio con los recursos educativos que producen, sin embargo de acuerdo a la infraestructura con que cuenten, muchas de ellas no pueden crear sus propios repositorios por lo cual los repositorios de recursos educativos que existen actualmente permiten la adición de REA a instituciones o público en general siempre y cuando cumplan con los estándares establecidos. 
Diseño de recurso educativo abierto con un sistema experto para la enseñanza-aprendizaje ...

Actualmente podemos encontrar repositorios de REA como: TEMOA [4] del Tecnológico de Monterrey, CLARISE [5] formado por instituciones educativas de nivel superior en Latinoamérica y RIUAMEX [6] propio de la Universidad Autónoma del Estado de México por citar algunos que ofrecen REA con información que posee autenticidad, validez y confiabilidad en diversas ramas del conocimiento.

\subsection{Sistemas Expertos}

La Inteligencia Artificial (IA) es una ciencia reciente en comparación con otras cuyo nombre se acuño en 1956 por Jonh McCarty. Abarca una gran cantidad de subcampos como la planificación autónoma, juegos, control autónomo, diagnosis, planificación logística, robótica, procesamiento de lenguaje y resolución de problemas, educación, enseñanza, sistemas tutores y sistemas expertos.

Los SE o sistemas basados en conocimiento, almacenan el conocimiento de expertos en un campo determinado. Para Peña [7] es un Sistema computacional que adquiere conocimiento especializado en un campo específico para explotarlo mediante métodos de razonamiento que emulan el desempeño del experto humano en la solución de problemas.

También puede definirse como un Sistema informático (Hardware y Software) que simula a los expertos humanos en un área de especialización dada [8].

Tabla 1. Clasificación general de Sistemas Expertos.

\begin{tabular}{ll}
\hline \multicolumn{1}{c}{ Por su configuración } & \multicolumn{1}{c}{ Por la tarea que ejecutan } \\
\hline & Sistemas Expertos de control. \\
& Sistemas Expertos de formación. \\
Sistemas Expertos Independientes. & Sistemas Expertos para diagnosis, \\
Sistemas Expertos híbridos. & depuración y reparación. \\
Sistemas Expertos dedicados. & Sistemas Expertos para supervisión. \\
Sistemas Expertos que presentan una & Sistemas Expertos de planificación. \\
arquitectura integrada. & Sistemas Expertos de diseño. \\
Sistemas Expertos embebidos. & Sistemas Expertos de pronóstico y \\
& predicción. \\
& Sistemas Expertos de interpretación. \\
\hline
\end{tabular}

Giarratano \& Riley mencionan que un Sistema Experto consta de [9]:

- Interfaz de usuario. Permite la comunicación entre el usuario y el Sistema experto.

- Medio de explicación. Explica al usuario el razonamiento del Sistema.

- Memoria activa. Contiene los hechos usados por las reglas.

- Mecanismo de inferencia. Infiere cuales reglas satisfacen los hechos u objetos ejecutando la prioridad más elevada de las reglas.

- Agenda. Creada por el mecanismo de inferencia y contiene una lista con prioridades asignadas a las reglas. 
- Medio para la adquisición de conocimiento (opcional). Permite introducir nuevo conocimiento al sistema por parte del usuario de manera automática.

Los SE se pueden clasificar de manera general como se muestra en la Tabla 1.

Pero ¿por qué usar sistemas expertos?, al dar respuesta a este cuestionamiento se identifica que los objetivos son variados ya que en el contexto sería difícil imaginar las necesidades para hacer uso de estos sistemas, sin embargo se pueden mencionar los siguientes:

1. Hay pocos expertos humanos y personal con poca experiencia, además de que resulta costosa la capacitación o es de un largo tiempo de preparación.

2. La combinación de conocimientos de varios expertos humanos daría como resultado sistemas expertos más confiables.

3. La agilidad de respuesta es rápida en problemas complicados.

4. El error humano está presente en los diversos problemas complejos por lo que la solución no puede ser fiable.

Dentro de los SE que más renombre han tenido desde su creación se puede mencionar [10]:

- MYCYN. Desarrollado en la Universidad de Stanford se encarga del diagnóstico de infecciones en la sangre y su terapia.

- MACSYMA. Trabaja con cálculo diferencial e integral.

- PROSPECTOR. Prospección y evaluación de yacimientos de minerales principalmente cobre y aluminio.

- DENDRAL. Analiza la estructura molecular de un compuesto.

- XCON. Primero en ser usado comercialmente para la configuración de los sistemas DEC/VAX.

\section{Trabajos relacionados}

La aplicación de los SE en la educación como se menciona en [11] puede hacer referencia a la aplicación de SE para el diseño de modelos instruccionales de enseñanza enfocado principalmente para el instructor que va a impartir el contenido y la otra aplicación enfocado al aprendizaje asistido por computadora como una herramienta que ayude al proceso enseñanza-aprendizaje de cualquier área del saber y que está enfocado a los alumnos quienes son los usuarios principales del SE.

Se han construido diversos SE que como se menciona en [12] los cuales la mayoría de ellos son prototipos. Se puede citar a SOPHIE y sus respectivas versiones SOPHIE II y SOPHIE III creado por la compañía Bolt, Beranek \& Newman que se diseñó para ejercitar la detección y reparación de averías en circuitos electrónicos. SCHOLAR es otro ejemplo, fue uno de los primeros sistemas que modeló el conocimiento procedural en forma de red semántica.

Actualmente existen SE de uso comercial [13] que se utilizan como herramientas para hallar resultados, programar o investigar con la simulación matemática. Los sistemas más conocidos tenemos Mathematica, Matlab y Geometer's Sketchpad. 
Mathematica [14] es un sistema que permite aprender, hacer y aplicar la matemática en diversas áreas del conocimiento por medio de cálculos en forma simbólica o numérica y de este modo resolver problemas con cualquier grado de dificultad.

Matlab es una herramienta de cálculo simbólico ofreciendo la funcionalidad de una súper calculadora y un intérprete de un lenguaje de programación propio por lo que es utilizado para el aprendizaje automático.

Geometer's Sketchpad [13] fue diseñado principalmente para servir como herramienta en el proceso de enseñanza-aprendizaje, es producido por Key Curriculum Press y fue desarrollado para la enseñanza de la geometría en la educación secundaria, en la práctica se ha visto que es una herramienta pedagógica que se puede utilizar en niveles superiores. Permite diseñar experiencias de aprendizaje en áreas como: la teoría de funciones, cálculo y ecuaciones diferenciales.

Existen otros tipos de SE que sólo son proyectos de investigación académico y tienen un objetivo particular. Se puede mencionar AgentGeom [15] desarrollado por Pedro Cobo y Josep M. Fortuny que es un Sistema Tutorial Inteligente que cuenta con una base de conocimientos definido por sus autores "espacio básico de un problema" que contiene todas las formas posibles de resolver un problema por parte de un experto. El sistema recibe las acciones por parte del alumno que son el dibujo de gráficas y las deducciones para la posible solución de un problema y al mismo tiempo el sistema le va indicando al alumno si lo que está realizando está mal por medio de mensajes de error por lo que el objetivo del sistema es ayudar directamente al alumno en la resolución del problema. La interfaz dedicada para el profesor permite asignar los problemas a sus alumnos o crear problemas nuevos.

\section{Materiales y métodos}

El diseño de los REA se basa en una metodología que indica los pasos a seguir en este caso el Modelo tecnológico para la creación de un Recurso Educativo Abierto (REA) como dice Ramírez [16] apoya como guía para la solución de problemas y se utiliza como auxiliar en las decisiones y la elaboración del diseño instruccional. Aquí se menciona la parte tecnológica, es necesario interactuar con la tecnología. Siguiendo los pasos de la metodología y el análisis de diferentes diseños instruccionales se puede realizar el diseño instruccional para la creación de los REA. El Modelo tecnológico consta de 5 etapas que se describen brevemente a continuación:

- Análisis. Planeación o secuencia didáctica.

- Diseño. Prototipo, evaluaciones del temario y evaluaciones del REA.

- Producción. Verificación de acuerdo a la planeación y al prototipo del diseño, consulta del catálogo de software multimedia, sugerencias de páginas de música o imágenes abiertas, decisión e inicio de la parte multimedia, realizar cambios de acuerdo a la sugerencia del docente.

- Desarrollo. Integración de prototipos, consulta de catálogo de software para realizar el REA, se documenta cada parte del REA, consultar y establecer metadatos.

- Implementación. Autoría, consultar catálogo de licenciamiento creative commons, validación, retroalimentación. 
Al ser un recurso compartido y de uso libre se trabaja con herramientas Open Source. Para el caso del diseño del prototipo se utiliza Evolus Pencil [17], para el desarrollo del REA como recurso Web se utiliza la herramienta eXeLearning [18] que es una herramienta de autor que sirve de apoyo a los docentes en la creación de contenidos Web, para las pruebas se utiliza Google Drive como servidor alojando el recurso.

Los SE de manera general están compuestos de los elementos que se muestran en la arquitectura de la Fig. 1.

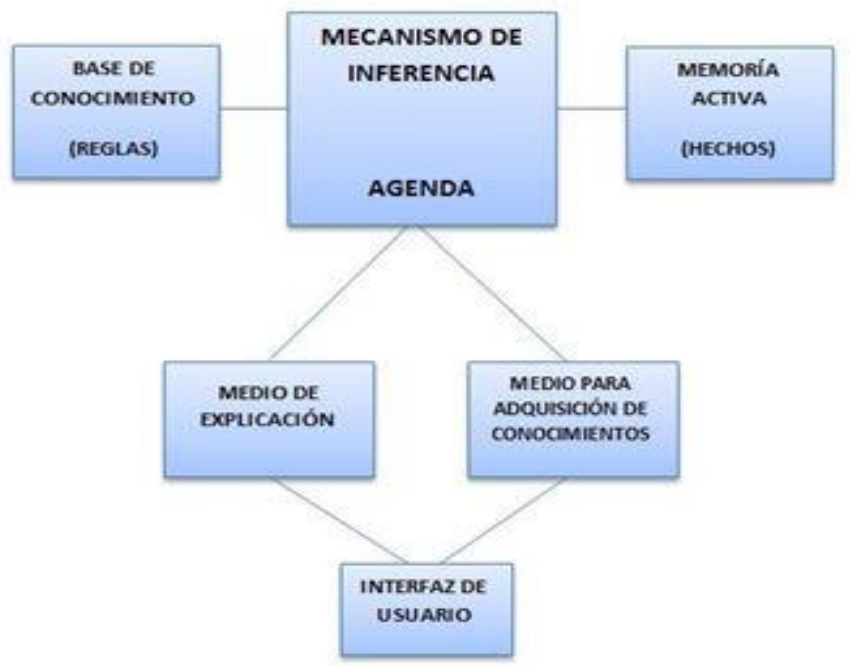

Fig. 1. Elementos de un sistema experto [9].

El diseño de un Sistema Experto se puede guiar de la arquitectura general dependiendo de la funcionalidad que se requiera.

También se puede hacer uso de alguna metodología para el diseño de sistemas, sin embargo se considera para este trabajo usar el Modelo lineal del ciclo de vida para desarrollar Sistemas Expertos, este modelo permite que solo se lleven a cabo aquellas tareas necesarias en cada etapa, por lo tanto no es obligatorio cubrir todas las tareas marcadas. La Fig. 2 muestra los pasos de esta metodología.

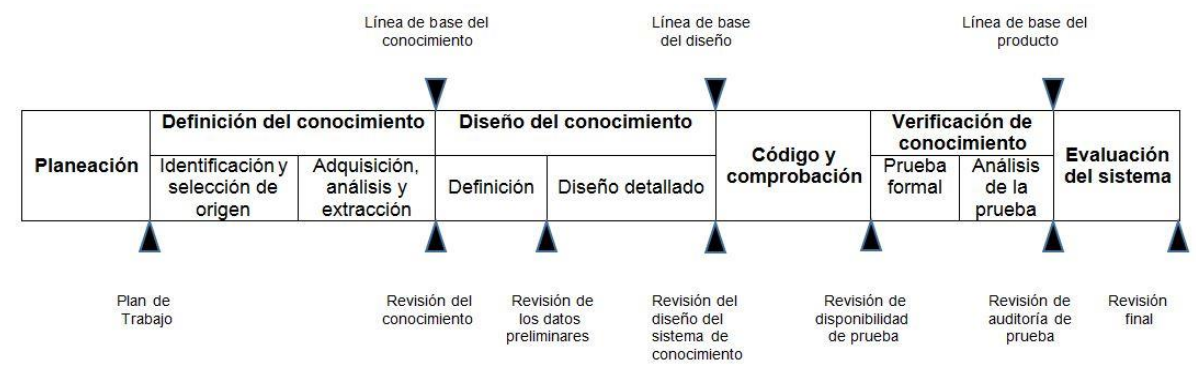

Fig. 2. Etapas del Modelo lineal del ciclo de vida para desarrollar sistemas expertos [9]. 
Diseño de recurso educativo abierto con un sistema experto para la enseñanza-aprendizaje ...

En la codificación del SE se hace uso de un lenguaje de programación lógico y siguiendo la línea del uso de herramientas Open Source se utiliza el programa SWIProlog [19] que permite crear sistemas basados en reglas y hechos.

En cuanto a la etapa de Definición del conocimiento, el origen del conocimiento se hace a través de libros, revistas, páginas de Internet, que se considera fueron creados por expertos, sin embargo, se pide el apoyo de un docente experto que imparte la materia de Base de Datos en el Nivel Medio Superior para presentar y explicar de una manera fácil y sencilla a los alumnos el contenido del REA y de las preguntas que se encuentran en el SE.

\section{Resultados}

El REA propuesto está diseñado para la enseñanza de los Fundamentos de bases de datos en el Nivel Medio Superior basado en el plan de estudios del bachillerato de la UAEM de la materia Informática Administrativa [20]. Dicho REA cuenta con autoevaluaciones al término de cada tema, además se pretende que al finalizar todo el curso el alumno pueda accesar a una evaluación final, donde esta fase del trabajo es a través de un SE. Está desarrollado en eXeLearning por lo que se muestra como una página web que cuenta con un menú mostrando todos los temas del módulo, referencias y material de apoyo, es en este último apartado donde se encuentra como archivo a descargar el SE el cual se muestra como archivo setup conteniendo todo lo necesario para instalarse en los equipos que utilicen los alumnos sin necesidad de tener instalado SWI-Prolog para poder trabajarlo. El SE se instala siguiendo un típico asistente de instalación y queda agregado como un programa más para su uso.

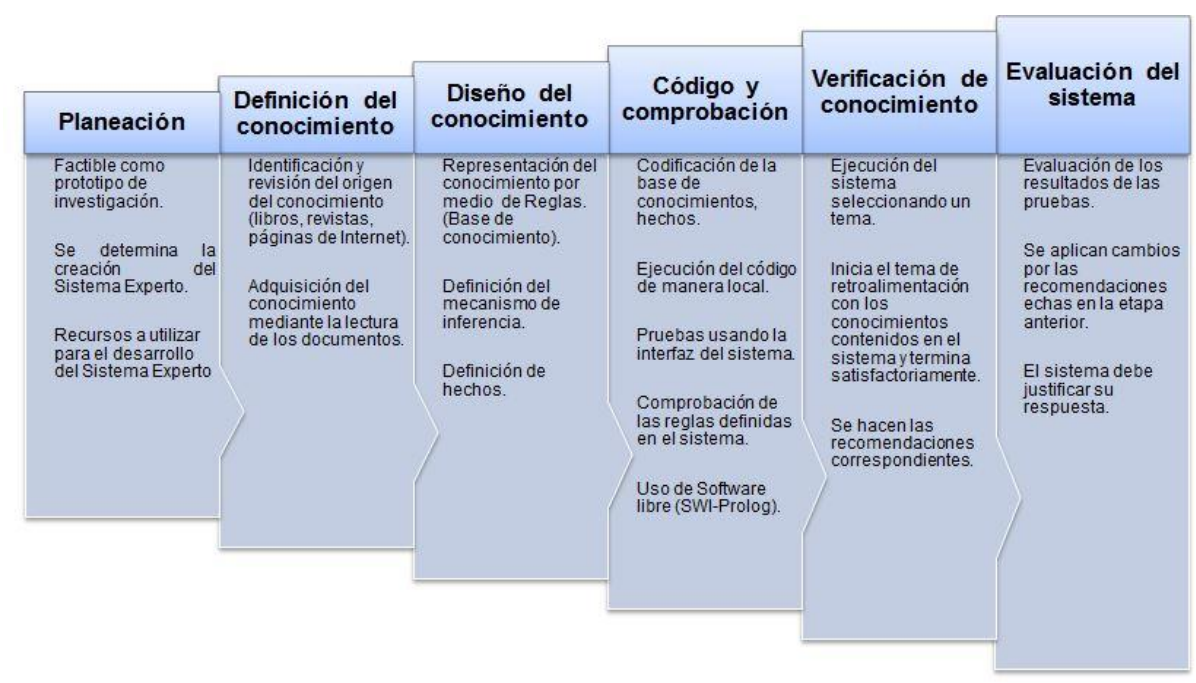

Fig. 3. Etapas del SE con el Modelo lineal del ciclo de vida para desarrollar sistemas expertos.

El SE propuesto se encuentra en fase de prototipo y está diseñado para el REA antes mencionado y se considera de tipo formación, ya que posee un base de conocimientos 
que se empleara en el adiestramiento de los alumnos y poder detectar las lagunas de conocimiento que el alumno padezca como conceptos o ideas equivocadas asimiladas con anterioridad. Por tal motivo, se le da al alumno una retroalimentación de lo visto en el recurso a través de una serie de preguntas. El modelado del SE está basado en el Modelo lineal del ciclo de vida para desarrollar sistemas expertos, en la Fig. 3 se puede observar brevemente lo realizado en cada una de las etapas del modelo en el SE.

Como se mencionó en el punto anterior la retroalimentación que ofrece el SE es con una serie de preguntas clasificadas por los temas principales que contiene el REA, que en este caso son 3: Introducción, modelo de datos y bases de datos relacionales que se definen dentro del código del Sistema como reglas Fig. 4.

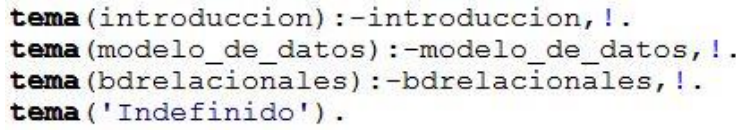

Fig. 4. Reglas establecidas en el SE para la selección del tema a repasar.

Cuando se ejecuta el SE pregunta cuál de los temas desea revisar para la retroalimentación Fig. 5.

\begin{tabular}{l}
\hline exa \\
Responde las siguientes preguntas \\
¿Quieres revisar el tema de: Definición, proposito y aplicación de una BD? \\
\hline
\end{tabular}

Fig. 5. Ventana que pregunta al alumno por el tema a revisar para la retroalimentación.

Cada uno de los temas tiene una base de hechos formado por las preguntas a realizar como se muestra a continuación:

introducción:-tema_introducción,

pregunta(‘¿Quieres revisar el tema de: Definición, propósito y aplicación de un BD?'),

pregunta('Un dato puede ser cualquier símbolo, número o letra que no tiene ningún significado.'),

pregunta('Un registro es un conjunto de campos con datos o información'),...

modelo_de_datos:-tema_modelo_de_datos,

pregunta(‘¿Quieres revisar el tema de: Modelo de datos?’),

pregunta('Los modelos de datos son herramientas que permiten modelar los datos y obtener un modelo de la realidad'),...

bdrelacionales:-tema_bdrelacionales,

pregunta(‘¿Quieres revisar el tema de: Base de datos relacionales?’), 
pregunta('Las tablas son objetos fundamentales de este tipo de BD y están formados por registros y campos.'),

Durante la retroalimentación el SE va verificando que el alumno conteste correctamente la mayoría de las preguntas, en caso contrario el SE corta la retroalimentación indicándole que es indefinido el aprendizaje que obtuvo, para esto se ocupa la regla 4 que se muestra como indefinido en la Fig. 4.

\section{Conclusiones y trabajo a futuro}

La educación en la actualidad exige docentes bien preparados y capacitados, además de que en la mayoría de las instituciones no se asignan de acuerdo a sus perfiles a las materias impartidas, debido a dichas exigencias y limitaciones.

Por una parte con la creación del REA de Fundamentos de bases de datos se obtiene un recurso con información valida y confiable que apoya la educación de los alumnos y la labor de enseñanza de los docentes.

El SE que se propone como se mencionó anteriormente se encuentra en fase de prototipo por lo cual aún no se ha evaluado el impacto, sin embargo se pretende realizar una prueba piloto con un grupo de Educación Media Superior que cursan la materia de Base de Datos y por medio de esta prueba realizar las evaluaciones correspondientes que permitan mejorar el SE en cuanto a su accesibilidad, comprensión, uso y que su contenido sea el ideal con respecto al REA.

La falta de expertos humanos en el ámbito educativo hace factible la necesidad de trabajar con SE que refuercen el aprendizaje de los alumnos proporcionándoles una herramienta que pueden trabajar en la escuela, en la casa o en algún lugar donde tengan acceso a una computadora teniendo la certeza que obtendrán una retroalimentación veraz y confiable, como si estuvieran de manera presencial con el docente.

Actualmente con toda la apertura tecnológica y de recursos educativos en línea se ha suscitado una revolución de enseñanza-aprendizaje virtual, generando infinidad de información que no está revisada ni estandarizada por lo que la elaboración de REA y SE permite ofrecer una educación de calidad con el uso de las herramientas apropiadas.

A largo plazo como dice Matkin [21] las instituciones educativas deben desarrollar y mantener repositorios abiertos de recursos educativos para compartir y reutilizar material educativo, por la misma razón las instituciones también debe generar sus propios REA y fomentar el uso de estos recursos en los docentes, alumnos, y personal que labora en la institución así como en otras instituciones.

Como se puede observar al final el SE se puede considerar un REA, por lo cual se puede centrar el trabajo en el desarrollo de SE como recurso total e individual, con los estándares necesarios para cubrir dicho objetivo.

El SE con los cambios adecuados puede evaluar al alumno, con lo que el docente tendría una evidencia de que realmente se está usando el SE además de que también conocería el nivel de aprovechamiento en cuanto a aprendizaje que ha estado obteniendo el alumno.

Agradecimientos. Los autores agradecen a la Universidad Autónoma del Estado de México y el autor principal agradece al CONACYT por el apoyo otorgado. 


\section{Referencias}

1. UNESCO. Comunicación e Información. Recursos Educativos Abiertos, http://www.unesco.org/new/es/communication-and-information/access-toknowledge/open-educational-resources/

2. Mortera, G.F.J., Salazar, R.A.L., Rodríguez, G.J., Pérez, N.J.A.: Guía de Referencia para el uso de Recursos Educativos Abiertos [REA] y Objetos de Aprendizaje [OA]. México: CUDI-CONACYT (2011)

3. Celaya, R.R., Lozano, M.F., Ramírez, M.M.S.: Apropiación tecnológica en profesores que incorporan recursos educativos abiertos en educación media superior. RMIE Revista Mexicana de investigación educativa, Vol. 45, pp. 213-487 (2010)

4. TEMOA. Portal de Recursos Educativos Abiertos, http://www.temoa.info/es

5. CLARISE. Comunidad Latinoamericana Abierta Regional de Investigación Social y Educativa, https://sites.google.com/site/redclarise/

6. RI UAEMEX, http://ri.uaemex.mx/

7. Peña, A.A.: Sistemas basados en conocimiento: Una Base para su Concepción y Desarrollo. Instituto Politécnico Nacional México (2006)

8. Castillo, E., Gutiérrez, J.M., Hadi, A.S.: Sistemas Expertos y Modelos Probabilísticos. España (s.f.)

9. Giarratano, J., Riley, G.: Sistemas Expertos: Principios y programación. International Thomson Editores México (2001)

10. Inteligencia Artificial. Extracción de ejemplos de Sistemas Expertos. Universidad Nacional Autónoma de México. http://www.ptolomeo.unam.mx:8080/xmlui/bitstream/ handle/132.248.52.100/219/A7.pdf?sequence=7

11. Montiel, L., Riveros, V.: Los sistemas expertos en el ámbito educativo. Omnia, pp. 11-28 (2014)

12. Amador, H.L.: Inteligencia artificial y sistemas expertos. Servicio de Publicaciones de la Universidad de Córdoba (1997)

13. Vílchez, Q.E.: Sistemas Expertos para la enseñanza y el aprendizaje de la matemática en la educación superior. Año 2, No. 3, pp. 45-67. Cuadernos de investigación y formación en educación matemática (2007)

14. UNAM-Wolfram Mathematica.: Preguntas frecuentes, http://www.software.unam.mx/ preguntas.html

15. Cobo, P., Fortuny, J.M.: AgentGeom: un sistema tutorial para el desarrollo de competencias argumentativas de los alumnos a través de la resolución de problemas. Matematicalia revista digital de divulgación matemática, Vol. 3, No. 3 (2007)

16. Ramírez, V.L.A.: Modelo tecnológico para la creación de un recurso educativo abierto (REA). Universidad Autónoma del Estado de México (2014)

17. Pencil Project. An open-source GUI prototyping tool, http://pencil.evolus.vn/

18. eXeLearning.net. El Nuevo eXeLearning...http://exelearning.net/

19. SWI-Prolog, http://www.swi-prolog.org/

20. UAEM Bachillerato Universitario: Programa de la Asignatura: Informática Administrativa, sexto semestre, http://denms.uaemex.mx/programas/

21. Matkin, G.W.: The Distance Educator's Opportunity for Institutional Leadership. Continuing Higher Education Review. University of California, Irvine CA, Vol. 74 (2010) 